\title{
Expression and immune characterization of a novel enzyme, protein arginine methyltransferase 1 , from Schistosoma japonicum
}

\author{
Wei Diao • Hejun Zhou • Wei Pan • Haipeng Liu • \\ Yujuan Shen $\cdot$ Yuxin Xu $\cdot$ Xiaohong Li $\cdot$ Jianping Cao
}

Received: 15 September 2013 / Accepted: 27 November 2013 /Published online: 17 December 2013

(C) The Author(s) 2013. This article is published with open access at Springerlink.com

\begin{abstract}
Protein arginine methyltransferase 1 (PRMT1) is an arginine-specific protein methyltransferase that methylates a number of proteins involved in transcription and RNA metabolism in all parasitic helminths, including the human blood fluke, Schistosoma japonicum. To characterize the role of PRMT1 in the development of S. japonicum and to investigate its influence on parasite-host interactions, we cloned and expressed the protein from an existing cDNA library. We report that the clone encoded a polypeptide comprising 360 amino acids with a predictive $\mathrm{Mr}$ of $42 \mathrm{kDa}$. Bioinformatic analyses predicted that there were many potential B cell epitopes and T cell epitopes associated with SjcPRMT1, suggesting it is a potential candidate molecule for vaccine development. The purified recombinant protein of $S$. japonicum (Chinese strain) (rSjcPRMT1) was found to be immunogenic, eliciting a high antibody titer in mice. Moreover, Western blot analysis revealed that the protein could be recognized by the sera of infected mice. Using flow cytometry, we showed that rSjcPRMT1 slightly upregulated the expression of CD40, CD80, CD86, and MHC-II molecules of mouse bone marrow-derived dendritic cell (BMDC), indicating that rSjcPRMT1 could induce mouse BMDC to mature and, therefore, activate their immune response. Overall, our findings provide evidence that $\mathrm{rSjcPRMT} 1$ could serve as an effective candidate molecule for the development of a vaccine against infection with $S$. japonicum .
\end{abstract}

W. Diao $\cdot$ H. Zhou $\cdot$ W. Pan $\cdot$ H. Liu $\cdot$ Y. Shen $\cdot$ Y. Xu $\cdot$ X. Li $\cdot$ J. Cao $(\square)$

Key Laboratory of Parasite and Vector Biology, Ministry of Health, WHO Collaborating Center for Malaria, Schistosomiasis and Filariasis, National Institute of Parasitic Diseases, Chinese Center for Disease Control and Prevention, Rui Jin Er Lu 207,

Shanghai 200025, People's Republic of China

e-mail: caojp@yahoo.com

\section{Introduction}

Schistosomiasis is a widespread infectious disease that constitutes a serious public health problem worldwide. While significant progress has been made in treatment of the disease with safer and more effective drugs now available, treatment does not prevent reinfection (Patz et al. 2000; McManus and Loukas 2008). Moreover, reinfection rates are high, which results in continuing outbreaks of high prevalence and high associated morbidity (McManus 2005). Vaccination offers an effective alternative to the treatment of schistosomiasis. However, to date, vaccines have not been used in clinical trials because their protective efficiency remains low (Xin et al. 2006; Siddiqui et al. 2011). Hence, there is an urgent need to identify and develop candidate molecules for use in future vaccines to protect again schistosomiasis. The reed vole, Microtus fortis (herein referred to as Mf), is the only known mammalian host in which schistosomes of Schistosoma japonicum are unable to mature, and thus, cause significant pathogenesis in hosts (Liu et al. 2001; Sun et al. 2004; Peng et al. 2011). Therefore, investigating the immunogenetic response of $\mathrm{Mf}$ to schistosome infection could help to identify new candidate vaccine molecules that protect this species from the debilitating effects of schistosomiasis.

Previously, we constructed and screened the cDNA library of schistosomu using the serum of wild-type Mf and identified 26 candidate genes likely involved in the immune response of Mf, including protein arginine methyltransferase 1 (PRMT1), high mobility group box-1(HMGB1), cytochrome b5, mitochondrion coding region, $16 \mathrm{~S}$. japonicum proteins of unknown function, and six novel encoded proteins (data not shown). Protein arginine methyltransferase 1 (PRMT1) is a type I enzyme that catalyzes the transfer of an S-adenosyl-Lmethionine to a broad spectrum of substrates, including histones, RNA-transporting proteins, and nuclear hormone 
receptor coactivators (Chiou et al. 2007). Protein arginine methylation is also involved in regulating various cellular processes including transcription regulation, DNA repair, RNA processing, and signal transduction (Bedford and Richard 2005; Hung et al. 2004; Krause et al. 2007). Hence, it is likely that this molecule could prove effective in the development of a vaccine against schistosomiasis. However, its role in host immune regulation following infection with $S$. japonicum infection remains elusive. In the present study, we aimed to characterize the role of PRMT1 in the development of S. japonicum and its influence on parasite-host interactions. To do so, we cloned and expressed the protein PRMT1 of S. japonicum (Chinese strain) (rSjcRPMI1) from our existing cDNA library and investigated its effects on immune responses in this host species.

\section{Materials and methods}

Ethics statement

This study was carried out in strict accordance with the recommendations in the Guide for the Care and Use of Laboratory Animals of the National Institute of Parasitic Diseases, Chinese Center for Disease Control and Prevention. The protocol was approved by the Laboratory Animal Welfare \& Ethics Committee (LAWEC), National Institute of Parasitic Diseases, Chinese Center for Diseases Control and Prevention (permit number: IPD 2011-006). All surgery was performed under sodium pentobarbital anesthesia, and all efforts were made to minimize suffering.

Amplification and cloning the gene encoding the target protein

RNA was isolated from adult S. japonicum (Chinese strain) via digestion, ligation, and sequencing. The gene fragment (SjcRPMI1) was then amplified by reverse transcription PCR (RT-PCR) using the total RNA as a template. The specific primers (sense primer: 5'-GCA GGA TCC ATG AAC GTT AAA AAT GGA GAA GC-3' and antisense primer: 5'-CGA CTC GAG TCA GCG CAT GCG ATA ATT AAA C-3', which included a $B a m \mathrm{H}$ I site and a Xho I site, respectively) were designed according to the electronic elongation sequence of SjcPRMT1. The amplification profile used was as follows: $95^{\circ} \mathrm{C}$ for $5 \mathrm{~min}$, followed by 35 cycles at $95^{\circ} \mathrm{C} / 45 \mathrm{~s}, 63^{\circ} \mathrm{C} /$ $45 \mathrm{~s}$, and $72{ }^{\circ} \mathrm{C} / 1 \mathrm{~min} 20 \mathrm{~s}$ and $72{ }^{\circ} \mathrm{C} / 5 \mathrm{~min}$ (final extension). PCR experiments were performed on a programmable Easterwin PCR system using Taq polymerase (Promega, America), and the PCR products were separated on $1.0 \%$ agarose gels and visualized under UV light by ethidium bromide staining. The PCR products were extracted from the agarose gel then double-digested with Bam $\mathrm{H}$ I and Xho I endonucleases and cloned into the prokaryotic expression vector pET28a (Biotec, Beijing, China). Following this the recombinant plasmid, pET28a-SjcPRMT1 was isolated and confirmed by DNA sequencing.

Prokaryotic expression and purification of the target protein

This plasmid was then transformed into Escherichia coli BL21 (DE3) (Biotec, Beijing, China). SjcPRMT1 was expressed as a 6His-SjcPRMT1 (His-SjcPRMT1) fusion protein upon isopropyl-D-thiogalactopyranoside induction $(1 \mathrm{mg} / \mathrm{mL}$ for $6 \mathrm{~h}$ ). The recombinant protein was purified by affinity column chromatography using a HISTrap ${ }^{\mathrm{TM}} \mathrm{FF}$ resin column (GE Healthcare, Milwaukee, WI, USA) followed by thrombin (Novagen) cleavage. Protein samples were fractionated using $15 \%$ sodium dodecyl sulfate polyacrylamide gel electrophoresis (SDS-PAGE) and visualized by Coomassie brilliant blue G-250 staining. The cleaved protein was excised from an SDSPAGE gel, and its identity was confirmed by mass spectrometry. The protein concentration was determined by the method of Bradford (1976).

\section{Western blotting analysis}

The purified recombinant His-SjcPRMT1 fusion protein was subjected to $15 \%$ SDS-PAGE, electrotransferred to nitrocellulose membrane (Osmonics), and probed with mouse antiSjcPRMT1 sera (1:1,500 dilution in phosphate-buffered saline [PBS]) as the primary antibody and horseradish peroxidaseconjugated rabbit anti-mouse immunoglobulin G (Sigma) as the secondary antibody. The signal was detected by an electrochemiluminescence Western blotting system (GE Healthcare/Amersham Biosciences). In addition, the purified recombinant SjcPRMT1 was probed with mouse serum (1:500) infected with the cercariae of S. japonicum .

\section{Dendritic cells stimulation and maturation}

Dendritic cells (DC) are the most potent antigen-presenting cells and play a major role in the initiation and regulation of the adaptive immune response to antigens. The solution of thrombin-cleaved SjcPRMT1 protein was filtered through a $0.22-\mu \mathrm{m}$ syringe filter (Acrodisc, Pall, Auckland, USA) and then applied to the Affinity Park ${ }^{\mathrm{TM}}$ Detoxi-gel TM Endotoxin Removing Gel column (Pierce, USA) to remove the endotoxin in the protein solution according to the manufacturer's instructions. This allowed the concentrations of SjcPRMT1 and the endotoxin in the elution to be determined. After being cultured for 8 days, the DCs derived from mouse bone marrow were incubated with $0.05,0.1,1$, or $10 \mu \mathrm{g} / \mathrm{mL}$ of $\mathrm{rSjcPRMT} 1$ for $24 \mathrm{~h}$. Lipopolysaccharide (LPS; $0.1 \mu \mathrm{g} / \mathrm{ml}$ ) and PBS were used to replace the protein as the positive and negative control, respectively. 
Phenotypic characterization of mouse bone marrow-derived DC after stimulation with PRMT 1

The expression of surface molecules MHC-II and CD11c on collected cells was analyzed using flow cytometry (Calibar BD, USA). To determine the differences of the expression of co-stimulation molecules of DCs, the CD11 ${ }^{+}$cells were purified using anti-CD11c antibodyconjugated beads (Miltenyi, Germany). They were then analyzed for the expression of CD40, CD80, CD86, and MHC-II.

At each step of the staining, $5 \times 10^{5}$ cells were stained with specific $\mathrm{Abs}$ for $30 \mathrm{~min}$ at $4{ }^{\circ} \mathrm{C}$ in $100 \mu \mathrm{L}$ of PBS containing $1 \%$ of bovine serum albumin (BSA) and $0.1 \%$ of sodium azide (PBS + BSA + NaN3). We used fluorescein isothiocyanate (FITC) or phycoerythrin (PE)labeled monoclonal Abs, including PE-conjugated $\mathrm{MHC}$ class II (I-Ab, mouse IgG2a, AF6-120.1), FITCconjugated CD11c (hamster IgG, HL3), PE-conjugated CD40 (hamster IgM, HM40-3), FITC-conjugated CD80 (rat IgG2a, 1G10), and FITC-conjugated CD86 (rat IgG2a, BL1). FITC- or PE-labeled mouse, rat, or hamster IgG was substituted for specific Abs for the negative controls. All Abs were purchased from Peprotech (England).

Gene Runner (Hastings Software, Inc.) was used to predict the secondary structure and the functional sites.

\section{Results}

Amplification and cloning of the gene encoding SjcPRMT1

RT-PCR amplification of SjcPRMT1 was successful with a single band revealed by agarose gel electrophoresis of approximately 1,100 bp size (Fig. 1a, lanes 1-3). This PCR product was cloned into the pET-28a vector to generate the pET-28aSjcPRMT1 recombinant plasmid (confirmed by DNA sequencing and double digestion with $B a m H$ I and Xho I; Fig. 1b, lanes 1-4).
Bioinformatics analysis of the target protein SjcPRMT1

Using a public analysis software in the website CBS (http:// www.cbs.dtu.dk/services/), a bioinformatic analysis of the SjcPRMT1 protein showed that its secondary structure contained numerous $\alpha$-helix, $\beta$-turn, and $\beta$-sheet structures, but no transmembrane structure (TM). Prosite motif research, meanwhile, showed that this protein carried seven protein kinase $\mathrm{C}$ phosphorylation sites, $3 \mathrm{~N}$-myristoylation sites, 6 casein kinase II phosphorylation sites, 1 Asn-glycosylation site, and 1 cAMP phosphorylation site. In addition, BepiPred 1.0 Server analysis (http://www.cbs.dtu.dk/services/BepiPred/) predicted the presence of many potential B cell epitopes and T cell epitopes in the SjcPRMT1 protein, suggesting it may be a good candidate for vaccine development.

Expression and Western blotting analysis

The recombinant SjcPRMT1 fusion protein was expressed in E. coli BL21 (DE3) and purified by affinity column chromatography. After dialysis, approximately $30 \mathrm{mg}$ of purified protein was obtained from $2,000 \mathrm{~mL}$ of culture. The final concentration of the protein was $0.5 \mathrm{mg} / \mathrm{mL}$ in PBS. SDSPAGE showed that the size of the purified fusion recombinant SjcPRMT1 was 42 kDa (Fig. 2a, lanes 4-7; Fig. 2b, lane 1). Results of the Western blotting analysis showed that both the anti-His-G HRP antibody and the sera from mouse infected with cercaria of $S$. japonicum reacted with the purified recombinant SjcPRMT1 protein (Fig. 2c, lanes 2 and 3), showing a band of approximately $42 \mathrm{kDa}$.

SjcPRMT1 upregulated the expression of MHC-II, CD40, CD80, and CD86 in mouse BMDC

LPS induced DC maturation with upregulation surface expression of MHC-II, CD40, CD80, and CD86 (van der Kleij et al. 2002; Martinez et al. 2007). This was used as the positive stimulation to evaluate the maturation of DCs incubated with SjcPRMT-1. The phenotype of treated DCs was analyzed by flow cytometry. rSjcPRMT-1 significantly activated DCs with
Fig. 1 Amplification and cloning of SjcPRMT1. a RT-PCR results of SjcPRMT1. The PCR product was approximately $1,100 \mathrm{bp}$ in size. $\mathbf{b}$ Identification of the recombinant plasmid of pET28aSjcPRMT1 by double digestion. The recombinant plasmid DNA was digested by Bam $\mathrm{H}$ I and Xho I M1, M2, M3, M4 are all DNA markers
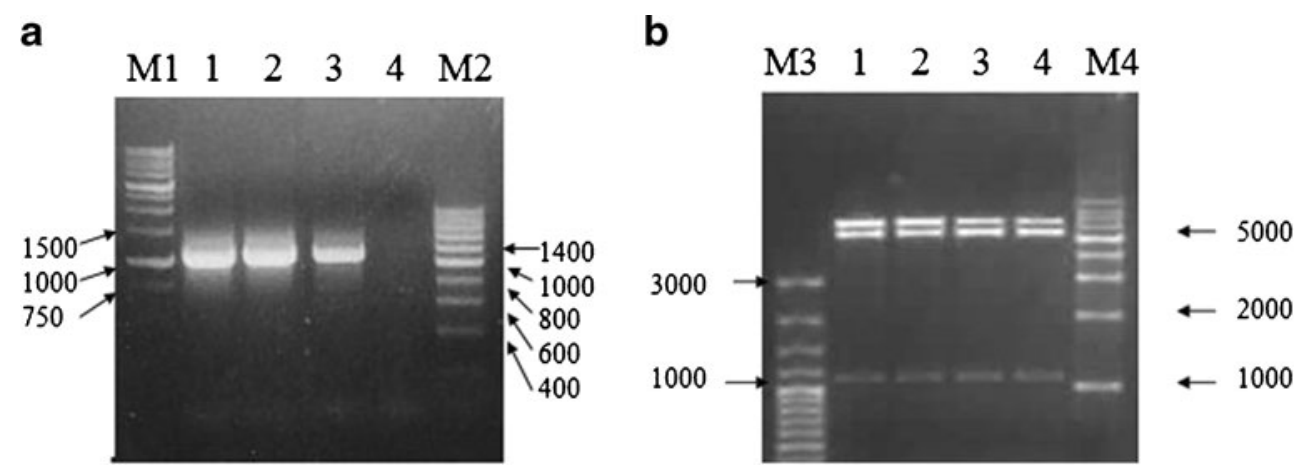
Fig. 2 Expression and identification of SjcPRMT1. a Analysis of recombinant SjcPRMT1 by SDS-PAGE. $M$ protein marker, 1 pET28a/BL21 before isopropyl $\beta$-D-1thiogalactopyranoside (IPTG) inducing, 2 pET28a/BL21 induced by IPTG after 4 h, 3 pET28a-SjcPRMT1/BL21 before IPTG inducing, 4-7 pET28aSjcPRMT1/BL21 induced by IPTG after 1, 2, 4, and $7 \mathrm{~h}$. b SDS-PAGE analysis of purified reSjcPRMT1. c Identification of reSjcPRMT1. $M$ protein marker, 1 normal mouse serum, 2 antiHis-G HRP antibody, 3 serum from mice infected with S. japonicum

a

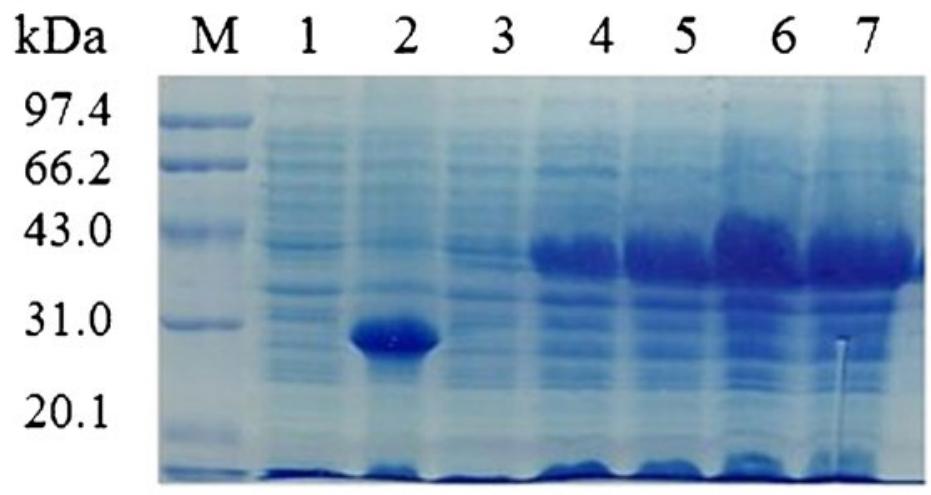

b

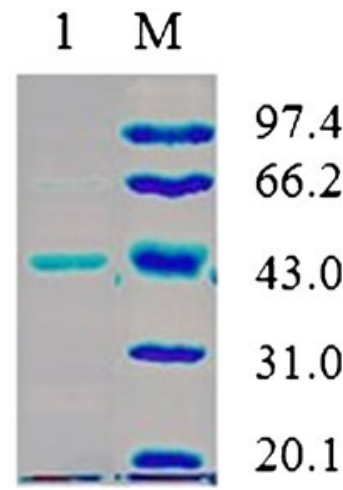

C

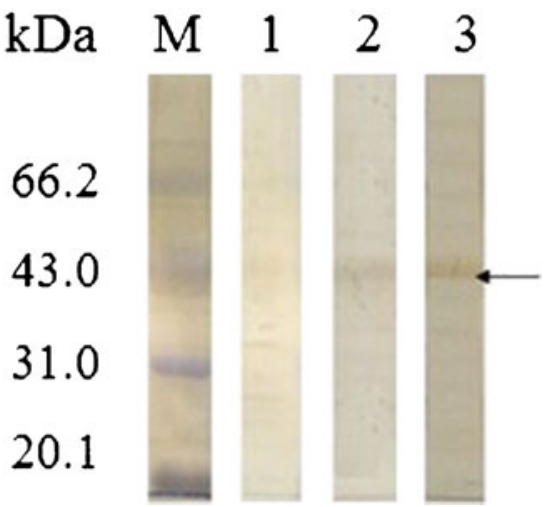

upregulation of MHC-II, CD40, CD80, and CD86 (Fig. 3), indicating that rSjcPRMT1 activated DCs and subsequently, $\mathrm{T}$ cells.

\section{Discussion}

Vaccination offers an effective alternative to the medical treatment of schistosomiasis, yet remains a poor therapeutic option due to the lack of efficacy of current vaccines (Xin et al. 2006; Siddiqui et al. 2011). Previously, we demonstrated that protein arginine methyltransferase 1 (PRMT1) plays a role in protecting the reed vole, $M$. fortis, from the negative physiological effects of $S$. japonicum infection. In this study, we successfully cloned and expressed the SjRPMI1 protein from our existing cDNA library and found that rSjcPRMI 1 may serve as an effective candidate molecule upon which to base the future development of a vaccine against infection with $S$. japonicum .

Protein arginine methyltransferase 1 (PRMT1), also known as HMT1 hnRNP methyltransferase-like 2, is an argininespecific protein methyltransferase that methylates a number of proteins involved in transcription and other aspects of RNA metabolism (Xu et al. 2003). It has been shown by three different research groups to be a protein that interacts with TIS21 and BTG1 in a yeast-2-hybrid screen (Lin et al. 1996), to interact with the interferon-alpha receptor (Abramovich et al.
1997), and to be homologous to the yeast homolog Hmtlp (Scott et al. 1998). PRMT1 is not required for adult viability but is required for differentiation during embryogenesis: mice lacking a functional PRMT1 gene die at an early stage of embryogenesis due to failure of the formation of the head and nervous system (Pawlak et al. 2000). The critical nature of PRMT1 in cellular function means that employing antibodies or vaccines to inhibit PRMT1 is likely to result in toxic effects.

In this study, we found that SjcPRMT 1 shared $87 \%$ homology to PRMT1 cloned from the closely related schistosome parasite, S. mansoni. This Sm PRMT1 protein has previously been demonstrated to methylate asymmetrically Arg-3 of histone $\mathrm{H} 4$ in vitro and to play a role in nuclear receptor-mediated chromatin remodeling and RNA transactions (Mansure et al. 2005). The similarity between Sj PRMT1 and Sm PRMT1 in terms of nucleotide and protein sequences, and the presence of an S-adenosylmethionine binding submotif, suggests that $\mathrm{Sj}$ PRMT1 is likely to be the homolog of Sm PRMT1. In addition to cloning and expression of $\mathrm{Sj}$ PRMT, we also obtained the purified rSj PRMT 1 protein from E. coli. Previous studies have shown that passive transfer of immunized sera among hosts also confers a degree of protection (Moloney et al. 1987); thus, it is assumed that antibodies in the immunized sera protect against $S$. japonicum infection. The result of our antigenicity assay showed that rSjcPRMT1 may be a potential candidate molecule for vaccine development, as it was successfully 

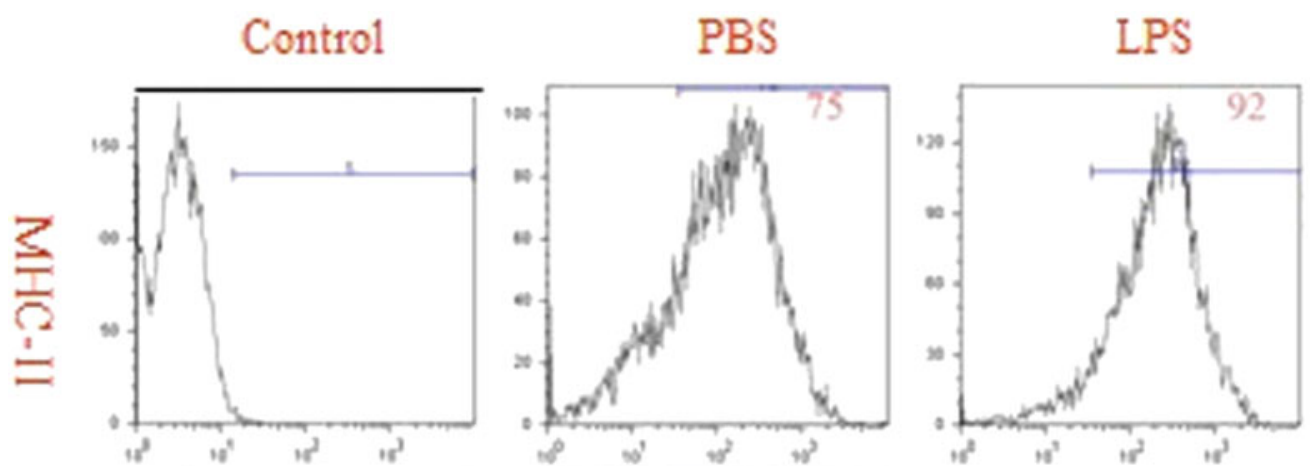

rSjcPRMT-1
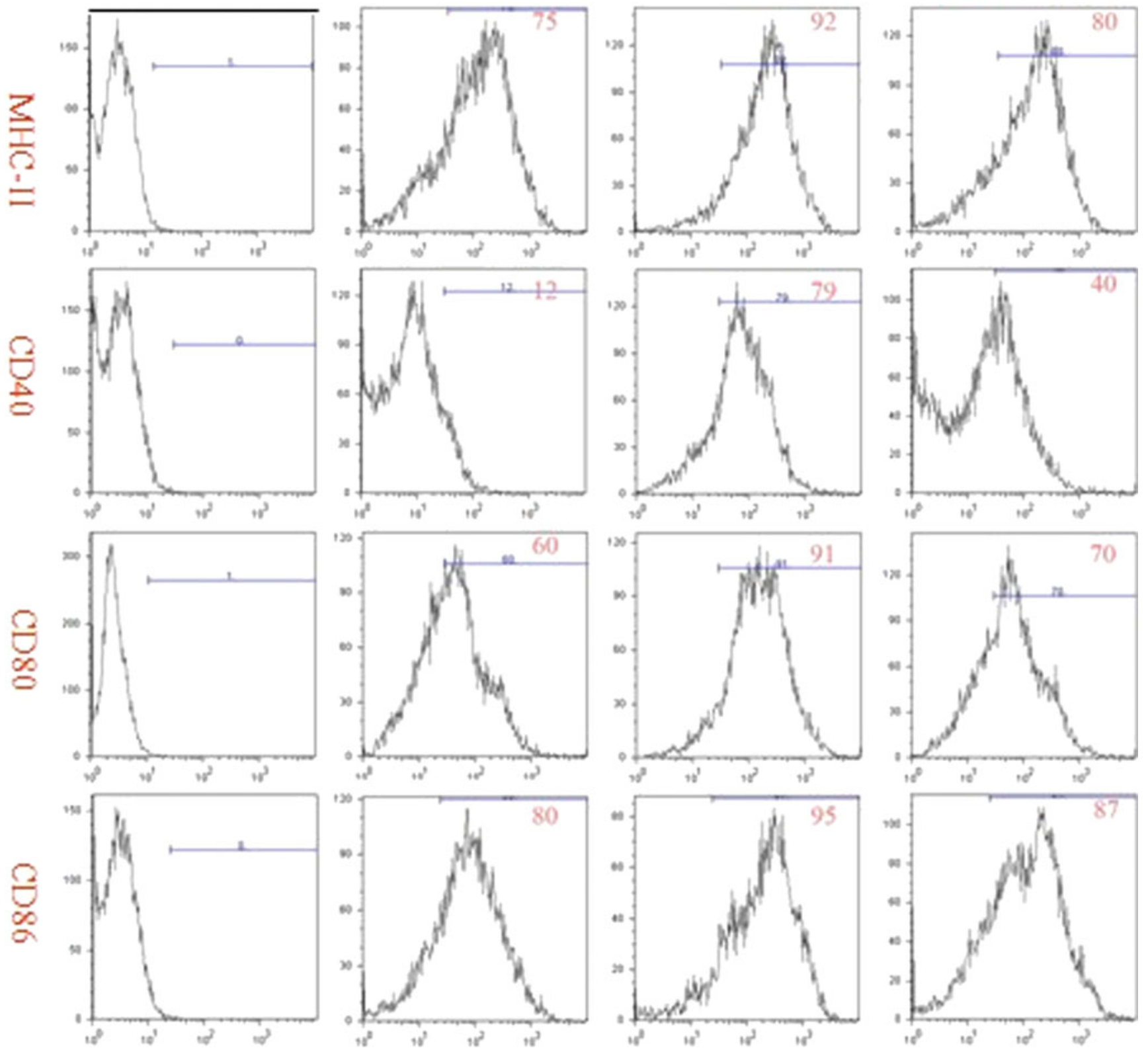

Fig. 3 Recombinant SjcPRMT1 regulated expression of surface markers of mouse BMDCs. CD11 ${ }^{+}$cells were purified using anti-CD11c antibody conjugated beads. The purified DCs with LPS or rSjcPRMT-1

stimulation were stained with indicated $\mathrm{mAb}$ and analyzed on an open gate for their phenotype by flow cytometry. Histograms represent one of the three flow cytometry experiments

probed by S. japonicum cercariae infected mouse serum. Han et al. (2010) evaluated the protection effects of recombinant PRMT1 against schistosomiasis japonica and found that the protein had a good ability to induce antibodies in mice, which resulted in worm burden reduced by $35.07 \%$ and egg burden reduced by $48.66 \%$.

PRMT1 gene was found to be expressed in the worm days 7, 13, 18, 23, 32, and 42 (Han et al. 2010) and especially a comparative higher expression in the adult of day 18. In the developmental stage of $S$. japonicum, male-female worm pairing begins at the days 17 to 18 , and then the female matured and spawned (Ross et al. 2001). During the period, the nutrition and energy metabolism of worm was especially required for providing related substances to maintain normal growth and eggs production. PRMT1 was therefore speculated to affect the nutrition and energy supplement in the development and maturation course of shistosomula.

Numerous studies have reported that DCs are the most potent antigen-presenting cells in the mammalian body and play a major role in the initiation and regulation of the adaptive immune response to pathogens [Martinez et al. 2007]. The function of these cells in initiating immunity is largely dependent on the 
expression of MHC-II, CD40, CD80, and CD86 on the surface of DCs themselves (Steinman 1991; Cella et al. 1997; Banchereau and Steinman 1998; Straw et al. 2003). In vivo, only mature DCs with high expression of these molecules can initiate immunity (Lutz and Schuler 2002). We have shown that rSjcPRMT 1 induces DCs to mature via the upregulation of MHC-II, CD40, CD80, and CD86 on DCs surface, which would initiate immunity in vivo. This property makes $\mathrm{rSj}$ PRMT 1 a potential antigen candidate, or adjuvant candidate, in the development of a new vaccine. Hence, the results of this study demonstrate that rSjcPRMT1 could induce mouse bone marrow-derived dendritic cell (BMDC) to mature and therefore activate host immune response. We suggest, therefore, that rSjcPRMI 1 may serve as an effective candidate molecule for the development of a vaccine against infection with $S$. japonicum .

Acknowledgments This work was supported by grants from the National Natural Science Foundation (nos. 81371841 and 30872212 to J.C., 30771880 to Y.S.) and the National S \& T Major Program (no. 2012ZX10004-201). The funders had no role in study design, data collection and analysis, decision to publish, or preparation of the manuscript.

Conflict of interests The authors have declared that no competing interests exist.

Open Access This article is distributed under the terms of the Creative Commons Attribution License which permits any use, distribution, and reproduction in any medium, provided the original author(s) and the source are credited.

\section{References}

Abramovich C, Yakobson B, Chebath J, Revel M (1997) A protein-arginine methyltransferase binds to the intracytoplasmic domain of the IFNAR1 chain in the type I interferon receptor. Embo J 16(2):260-266

Banchereau J, Steinman RM (1998) Dendritic cells and the control of immunity. Nature 392(6673):245-252

Bedford MT, Richard S (2005) Arginine methylation an emerging regulator of protein function. Mol Cell 18(3):263-272

Bradford M (1976) A rapid and sensitive methods for the quantitation of microgram quantities of protein utilizing the principle of protein-dye binding. Anal Biochem 72:248-254

Cella M, Engering A, Pinet V, Pieters J, Lanzavecchia A (1997) Inflammatory stimuli induce accumulation of MHC class II complexes on dendritic cells. Nature 388(6644):782-787

Chiou YY, Lin WJ, Fu SL, Lin CH (2007) Direct mass-spectrometric identification of Arg296 and Arg299 as the methylation sites of hnRNP K protein for methyltransferase PRMT1. Protein J 26(2):87-93

Han HX, Peng JB, Hong Y, Wang XZ, Shi YJ, Fu ZQ, Liu JM, Cheng GF, Li XR, Lin JJ (2010) Cloning, expression and immunoprotection of the novel gene SjPRMT1 of Schistosoma japonicum. Sci Agric Sin 43(4):835-841

Hung CM, Li C (2004) Identification and phylogenetic analyses of the protein arginine methyltransferase gene family in fish and ascidians. Gene 340(2):179-187

Krause CD, Yang ZH, Kim YS, Lee JH, Cook JR, Pestka S (2007) Protein arginine methyltransferases: evolution and assessment of their pharmacological and therapeutic potential. Pharmacol Ther 113(1):50-87

Lin WJ, Gary JD, Yang MC, Clarke S, Herschman HR (1996) The mammalian immediate-early TIS 21 protein and the leukemia- associated BTG1 protein interact with a protein-arginine $N$-methyltransferase. J Biol Chem 271(25):15034-15044

Liu JM, Fu ZQ, Li H, Wei MX, Lin JJ (2001) Prelinimary observation on the killing effect to shistosomula of Schistosoma japonicum in vitro by ADCC of Microtus fortis. Acta Parasitol Med Entomol Sin 8(4):212-219

Lutz MB, Schuler G (2002) Immature, semi-mature and fully mature dendritic cells: which signals induce tolerance or immunity. Trends Immunol 23(9):445-449

Mansure JJ, Furtado DR, de Oliveira FM, Rumjanek FD, Franco GR, Fantappie MR (2005) Cloning of a protein arginine methyltransferase PRMT1 homolog from Schistosoma mansoni: evidence for roles in nuclear receptor signaling and RNA metabolism. Biochem Biophys Res Commun 335(4):1163-1172

Martinez D, Vermeulen M, von Euw E, Sabatte J, Maggini J, Ceballos A, Trevani A, Nahmod K, Salamone G, Barrio M, Giordano M, Amigorena S, Geffner J (2007) Extracellular acidosis triggers the maturation of human dendritic cells and the production of IL-12. J Immunol 179(3):1950-1959

McManus DP (2005) Prospects for development of a transmission blocking vaccine against Schistosoma japonicum. Parasite Immunol 27(7-8):297-308

McManus DP, Loukas A (2008) Current status of vaccines for schistosomiasis. Clin Microbiol Rev 21(1):225-242

Moloney NA, Hinchcliffe P, Webbe G (1987) Passive transfer of resistance to mice with sera from rabbits, rats or mice vaccinated with ultraviolet-attenuated cercariae of Schistosoma japonicum. Parasitology 94(Pt 3):497-508

Patz JA, Graczyk TK, Geller N, Vittor AY (2000) Effects of environmental change on emerging parasitic diseases. Int J Parasitol 30(1213):1395-1405

Pawlak MR, Scherer CA, Chen J, Roshon MJ, Ruley HE (2000) Arginine $\mathrm{N}$-methyltransferase 1 is required for early postimplantation mouse development, but cells deficient in the enzyme are viable. Mol Cell Biol 20(13):4859-4869

Peng JB, Gobert GN, Hong Y, Jiang WB, Han HX, McManus DP, Wang XZ, Liu JM, Fu ZQ, Shi YJ, Lin JJ (2011) Apoptosis governs the elimination of Schistosoma japonicum from the non-permissive host Microtus fortis. PLoS ONE 6(6):e21109

Ross AG, Sleigh AC, Li Y, Davis GM, Williams GM, Jiang Z, Feng Z, McManus DP (2001) Schistosomiasis in the People's Republic of China: prospects and challenges for the 21 st century. Clin Microbiol Rev 14(2):270-295

Scott HS, Antonarakis SE, Lalioti MD, Rossier C, Silver PA, Henry MF (1998) Identification and characterization of two putative human arginine methyltransferases (HRMT1L1 and HRMT1L2). Genomics 48(3):330-340

Siddiqui AA, Siddiqui BA, Ganley-Leal L (2011) Schistosomiasis vaccines. Hum Vaccin 7(11):1192-1197

Steinman RM (1991) The dendritic cell system and its role in immunogenicity. Annu Rev Immunol 9:271-296

Straw AD, MacDonald AS, Denkers EY, Pearce EJ (2003) CD154 plays a central role in regulating dendritic cell activation during infections that induce Th1 or Th2 responses. J Immunol 170(2):727-734

Sun J, Lin JJ, Chen GF (2004) Gene difference expression between mice and Microtus fortis infected with Schistosoma japonicum using cDNA microarrays. Acta Sci Nat Univ Pekinensis 40(4):532-537

van der Kleij D, Latz E, Brouwers JF, Kruize YC, Schmitz M, Kurt-Jones EA, Espevik T, de Jong EC, Kapsenberg ML, Golenbock DT, Tielens AG, Yazdanbakhsh M (2002) A novel host-parasite lipid cross-talk. Schistosomal lyso-phosphatidylserine activates toll-like receptor 2 and affects immune polarization. J Biol Chem 277(50):48122-48129

Xin XF, Zhu YC, Wang ZG (2006) Progress of research on vaccines of schistosomiasis japonica. Chin J Schisto Control 18(5):397-399

Xu C, Henry PA, Setya A, Henry MF (2003) In vivo analysis of nucleolar proteins modified by the yeast arginine methyltransferase Hmt1/Rmt1p. Rna 9(6):746-759 